\title{
On Some Properties of Fuzzy Soft almost Soft Continuous Mappings
}

\author{
SABIR Hussain ${ }^{1, a}$
}

\begin{abstract}
In this paper, we initiate and explore the interesting characterizations and properties of fuzzy soft almost soft continuous mappings in fuzzy soft classes. We also study and discuss the notions of fuzzy soft almost soft open(closed) mappings. Moreover the characterizations of composition of two fuzzy soft almost soft mappings are also studied. We hope that the findings in this paper will be useful for the researchers working in the fields such as fuzzy control systems, fuzzy automata, fuzzy logic, information systems and decision making problems.

Key words and phrases. Fuzzy soft sets, Fuzzy soft topology, Fuzzy soft classes, Fuzzy soft almost soft continuous, fuzzy soft almost soft open(closed).
\end{abstract}

\section{Introduction}

Fuzzy set theory initiated and studied by Zadeh[31] proved to be an important mathematical tool to solve different types of complicated problems having uncertainties in real life problems such as sociology, economics, engineering, computer and medical sciences etc. with ambiguous environment. Now a days, both mathematician and computer scientists are applying this theory, not limited to such as fuzzy control systems, fuzzy automats, fuzzy logic, fuzzy topology etc.

Molodtsov [24] introduced the concept of soft sets, which is new approach for modelling the complicated problems with uncertainties. Molodtsov et. al[24] applied the technique of soft sets into several directions. Maji et. al[22] studied the several concepts of soft sets and applied soft sets in decision making problems. Many researchers like [7-10], [27] improved the work of Maji et. al. In [27], it is observed that there exists some compact connection between soft sets and information systems. Moreover, it is pointed out that the research work on soft sets and information systems can be unified. Maji and Biswas[23] generalized the soft sets and introduced fuzzy soft sets. After that many researchers such as Z. Kong et. al[21] studied the concept of fuzzy soft sets and presented the fuzzy

Received March 28, 2017 - Accepted May 14, 2018, published online in July 2018.

(C) The Author(s) 2017. This article is published with open access by Sidi Mohamed Ben Abdallah University.

1 Department of Mathematics, College of Science, Qassim University, P.O. Box 6644, Buraydah 51482, Saudi Arabia.

a e-mail: sabiriub@yahoo.com; sh.hussain@qu.edu.sa 
soft set theoretic approach to decision making problems.

Ahmad and Kharral[1] initiated the concept of mappings on fuzzy soft classes and introduce fuzzy soft images and fuzzy soft inverse images. The study of algebraic structures of soft sets and fuzzy soft sets have been increasing rapidly in recent years. Shabbir and Naz[28] defined and explored the concept of soft topological spaces. Further results, structures and improvements in concepts of soft topological spaces have been studied in [2], [7-9], [12-15], [25].

Hussain[16] continued to study the algebraic structures of soft semi-open(closed) sets in soft topological spaces initiated by Chen[4-5]. Chang[3] introduced and discussed the basic properties of fuzzy topological spaces. Tanay and Kandemir[29] studied the topological structures of fuzzy soft sets. Varol and Aygun[30] initiated fuzzy soft topology. Further structures of fuzzy soft topology are explored in [11], [20].

Recently, Hussain[17] initiated the concept of fuzzy soft semi-open sets as a generalization of soft semi-open sets and discussed its basic properties. In [18-19], Hussain studied and explored the weak and strong forms of fuzzy soft open sets as well as fuzzy soft semi-pre-open sets and fuzzy soft semi-pre-continuous mappings in fuzzy soft topological spaces. Hussain also developed the relationship between these newly defined concepts in [18-19].

\section{Preliminaries}

Definition 2.1[31]. A fuzzy set $f$ on $X$ is a mapping $f: X \rightarrow I=[0,1]$. The value $f(x)$ represents the degree of membership of $x \in X$ in the fuzzy set $f$, for $x \in X$.

Definition 2.2[24]. Let $X$ be an initial universe and $E$ be a set of parameters. Let $P(X)$ denotes the power set of $X$ and $A$ be a non-empty subset of $E$. A pair $(F, A)$ is called a soft set over $X$, where $F$ is a mapping given by $F: A \rightarrow P(X)$. In other words, a soft set over $X$ is a parameterized family of subsets of the universe $X$. For $e \in A$, $F(e)$ may be considered as the set of $e$-approximate elements of the soft set $(F, A)$.

Definition. 2.3[23]. Let $I^{X}$ denotes the set of all fuzzy sets on $X$ and $A \subseteq X$. A pair $(f, A)$ is called a fuzzy soft set over $X$, where $f: X \rightarrow I^{X}$ is a function. That is, for each $a \in A, f(a)=f_{a}: X \rightarrow I$, is a fuzzy set on $X$.

Definition 2.4[23]. For two fuzzy soft sets $(f, A)$ and $(g, B)$ over a common universe $X$, we say that $(f, A)$ is a fuzzy soft subset of $(g, B)$ if

(1) $A \subseteq B$ and

(2) for all $a \in A$ and $b \in B, f_{a} \leq g_{b}$; implies $f_{a}$ is a fuzzy subset of $g_{b}$.

We denote it by $(f, A) \tilde{\leq}(g, B)$. $(f, A)$ is said to be a fuzzy soft super set of $(g, B)$, if $(g, B)$ is a fuzzy soft subset of $(f, A)$. We denote it by $(f, A) \tilde{\geq}(g, B)$.

Definition 2.5[23]. Two fuzzy soft sets $(f, A)$ and $(g, B)$ over a common universe $X$ are said to be fuzzy soft equal, if $(f, A)$ is a fuzzy soft subset of $(g, B)$ and $(g, B)$ is a fuzzy soft subset of $(f, A)$.

Definition 2.6[23]. The union of two fuzzy soft sets of $(f, A)$ and $(g, B)$ over the common universe $X$ is the fuzzy soft set $(h, C)$, where $C=A \cup B$ and for all $c \in C$,

$$
h_{c}=\left\{\begin{array}{cc}
f_{c}, & \text { if } c \in A-B \\
g_{c}, & \text { if } c \in B-A \\
f_{c} \vee g_{c}, & \text { if } c \in A \cap B
\end{array}\right.
$$

We write $(f, A) \tilde{V}(g, B)=(h, C)$.

Definition 2.7[23]. The intersection $(h, C)$ of two fuzzy soft sets $(f, A)$ and $(g, B)$ over a common universe $X$, denoted $(f, A) \tilde{\wedge}(g, B)$, is defined as $C=A \cap B$, and $h_{c}=f_{c} \wedge g_{c}$, for all $c \in C$.

Definition 2.8[23]. The difference $(h, C)$ of two fuzzy soft sets $(f, A)$ and $(g, B)$ over $X$, denoted by $(f, A) \tilde{\backslash}(g, B)$, is defined as $(f, A) \tilde{\backslash}(g, B)=(f, A) \tilde{\wedge}(f, B)^{c}$.

For our convenience, we will use the notation $f_{A}$ for fuzzy soft set instead of $(f, A)$.

Definition 2.9[29]. Let $\tau$ be the collection of fuzzy soft sets over $X$, then $\tau$ is said to be a fuzzy soft topology on $X$, if

(1) $\tilde{0}_{A}, \tilde{1_{A}}$ belong to $\tau$.

(2) If $\left(f_{A}\right)_{i} \in \tau$, for all $i \in I$, then $\tilde{\bigvee}_{i \in I}\left(f_{A}\right)_{i} \in \tau$. 
(3) $f_{a}, g_{b} \in \tau$ implies that $f_{a} \tilde{\bigwedge} g_{b} \tilde{\in} \tau$.

The triplet $(X, \tau, A)$ is called a fuzzy soft topological space over $X$. Every member of $\tau$ is called fuzzy soft open set. A fuzzy soft set is called fuzzy soft closed if and only if its complement is fuzzy soft open.

Definition 2.10[30]. Let $(X, \tau, A)$ be a fuzzy soft topological space over $X$ and $f_{A}$ be a fuzzy soft set over $X$. Then

(1) fuzzy soft interior of fuzzy soft set $f_{A}$ over $X$ is denoted by $\left(f_{A}\right)^{\circ}$ and is defined as the union of all fuzzy soft open sets contained in $f_{A}$. Thus $\left(f_{A}\right)^{\circ}$ is the largest fuzzy soft open set contained in $f_{A}$.

(2) fuzzy soft closure of $f_{A}$, denoted by $\overline{f_{A}}$ is the intersection of all fuzzy soft closed super sets of $f_{A}$. Clearly $\overline{f_{A}}$ is the smallest fuzzy soft closed set over $\mathrm{X}$ which contains $f_{A}$.

Definition 2.11[17]. Let $(X, \tau, A)$ be a fuzzy soft topological space over $X$. A fuzzy soft set $f_{A}$ is called fuzzy soft semi-open, if there exists a fuzzy soft open set $g_{A}$ such that $g_{A} \tilde{\leq} f_{A} \tilde{\leq} \overline{g_{A}}$. The class of all fuzzy soft semi-open sets in $X$ is denoted by $\operatorname{FSSO}(\mathrm{X})$. Note that every fuzzy soft open set is fuzzy soft semi-open but the converse is not true in general.

Definition 2.12[17]. A fuzzy soft set $f_{A}$ in fuzzy soft topological space $(X, \tau, A)$ is fuzzy soft semi-closed if and only if its complement $\left(f_{A}\right)^{c}$ is fuzzy soft semi-open. The class of fuzzy soft semi-closed sets is denoted by $\mathrm{FSSC}(\mathrm{X})$. Note that every fuzzy soft closed set is fuzzy soft semi-closed in fuzzy soft topological space $(X, \tau, A)$.

Proposition 2.13[17]. Let $f_{A}$ be a fuzzy soft set in fuzzy soft topological space $(X, \tau, A)$. Then $f_{A}$ is fuzzy soft semi-closed if and only if there exists a fuzzy soft closed set $h_{A}$ such that $\left(h_{A}\right)^{0} \tilde{\leq} f_{A} \tilde{\leq} h_{A}$.

Definition 2.14[17]. Let $f_{A}$ be a fuzzy soft set in fuzzy soft topological space $(X, \tau, A)$. The fuzzy soft semi-closure of $f_{A}$, denoted by $s c l^{f s}\left(f_{A}\right)$ and is defined as the intersection of all fuzzy soft closed supersets of $f_{A}$.

It is clear from the definition that $s l^{f s}\left(f_{A}\right)$ is the smallest fuzzy soft semi-closed set over $X$ which contains $f_{A}$.

Definition 2.15[17]. Let $f_{A}$ be a fuzzy soft set in fuzzy soft topological space $(X, \tau, A)$. The fuzzy soft semi-interior of $f_{A}$, denoted by $\operatorname{sint} t^{f s}\left(f_{A}\right)$ and is defined as the union of all fuzzy soft open subsets of $f_{A}$.

It is clear from the definition that $\operatorname{sint} t^{f s}\left(f_{A}\right)$ is the largest fuzzy soft semi-open set over $X$ contained in $f_{A}$.

Definition 2.16[18]. Let $(X, \tau, A)$ be a fuzzy soft topological space over $X$. Then a fuzzy soft set $f_{A}$ over $X$ is said to be a fuzzy soft pre-open(respt. closed), if $f_{A} \tilde{\leq}\left(\overline{f_{A}}\right)^{\circ}\left(\right.$ respt. $\left.\overline{\left(f_{A}\right)^{\circ}} \tilde{\leq} f_{A}\right)$.

Definition 2.17[1]. Let $F(X, A)$ and $F(Y, B)$ be families of fuzzy soft sets. $u: X \rightarrow Y$ and $p: A \rightarrow B$ are mappings. Then a function $f_{p u}: F(X, A) \rightarrow F(Y, B)$ defined as :

(1) Let $f_{A}$ be a fuzzy soft set in $F(X, A)$. The image of $f_{A}$ under $f_{p u}$, written as $f_{p u}\left(f_{A}\right)$, is a fuzzy soft set in $F(Y, B)$ such that for $\beta \in p(A) \subseteq B$ and $y \in Y$,

$$
f_{p u}\left(f_{A}\right)(\beta)(y)=\left\{\begin{array}{cc}
\bigvee_{x \in u^{-1}(y)}\left(\bigvee_{\alpha \in p^{-1}(\beta) \cap A}\left(f_{A}(\alpha)\right),\right. & u^{-1}(y) \neq \phi, p^{-1}(\beta) \cap A \neq \phi \\
0, & \text { otherwise }
\end{array},\right.
$$

for all $y \in B . f_{p u}\left(f_{A}\right)$ is known as a fuzzy soft image of a fuzzy soft set $f_{A}$.

(2) Let $g_{B}$ be a fuzzy soft set in $F(Y, B)$. Then the inverse image of $g_{B}$ under $f_{p u}$, written as $f_{p u}^{-1}\left(g_{B}\right)$, is a fuzzy soft set in $F(X, A)$ such that

$$
f_{p u}^{-1}\left(g_{B}\right)(\alpha)(x)=\left\{\begin{array}{cc}
g(p(\alpha))(u(x)), & p(\alpha) \in B \\
0, & \text { otherwise }
\end{array},\right.
$$

for all $x \in A$. $f_{p u}^{-1}\left(g_{B}\right)$ is known as a fuzzy soft inverse image of a fuzzy soft set $g_{B}$.

The fuzzy soft function $f_{p u}$ is called fuzzy soft surjective, if $p$ and $u$ are surjective. The fuzzy soft function $f_{p u}$ is called fuzzy soft injective, if $p$ and $u$ are injective.

Definition 2.18[19]. Let $(X, \tau, A)$ be a fuzzy soft topological space over $X$, where $X$ is a nonempty set and $\tau$ is a family of fuzzy soft sets. Then a fuzzy soft set $f_{A}$ is said to be a fuzzy soft semi-pre-open, if there exists a fuzzy soft pre-open set $g_{A}$ such that $g_{A} \tilde{\leq} f_{A} \tilde{\leq} \overline{\left(g_{A}\right)}$.

Definition 2.19[19]. Let $(X, \tau, A)$ be a fuzzy soft topological space over $X$, where $X$ is a nonempty set and $\tau$ is a family of fuzzy soft sets. Then a fuzzy soft set $f_{A}$ is said to be a fuzzy soft semi-pre-closed, if there exists a fuzzy soft pre-closed set $g_{A}$ such that $\left(g_{A}\right)^{\circ} \tilde{\leq} f_{A} \tilde{\leq} g_{A}$.

Note that the fuzzy soft set $f_{A}$ is fuzzy soft semi-pre-open if and only if $f_{A}^{c}$ is fuzzy soft semi-pre-closed.

Remark 2.20[19]. It is clear that any fuzzy soft semi-open as well as fuzzy soft pre-open set is a fuzzy soft 
semi-pre-open set.

Definition 2.21[19]. Let $f_{A}$ be a fuzzy soft set in fuzzy soft topological space $(X, \tau, A)$ over $X$. Then

(1) fuzzy soft semi-pre-interior of fuzzy soft set $f_{A}$ denoted by $F^{s}$ pint $t^{s}$ and is defined as $F^{s}$ pint $t^{s}\left(f_{A}\right) \tilde{=} S u p\left\{g_{A}: g_{A}\right.$ is fuzzy soft semi-pre-open and $\left.g_{A} \tilde{\leq} f_{A}\right\}$.

(2) fuzzy soft semi-pre-closure of fuzzy soft set $f_{A}$ denoted by $F^{s} p c l^{s}$ and is defined as $F^{s} p c l^{s}\left(f_{A}\right) \stackrel{\sim}{=} \operatorname{In}\left\{g_{A}: g_{A}\right.$ is fuzzy soft semi-pre-closed and $\left.f_{A} \tilde{\leq} g_{A}\right\}$.

Definition 2.22[18]. A fuzzy soft set $f_{A}$ is said to be a fuzzy soft point in $(X, \tau, A)$ denoted by $e\left(f_{A}\right)$, if for the element $e \tilde{\in} A, f(e) \tilde{\neq} \tilde{0}$ and $f\left(e^{c}\right) \tilde{=} \tilde{0}$, for all $e^{c} \in A \backslash\{e\}$.

Definition 2.23[30]. Let $\left(X, \tau_{1}, A\right)$ and $\left(Y, \tau_{2}, B\right)$ are fuzzy soft topological spaces and $f_{p u}: F(X, A) \rightarrow F(Y, B)$ be a fuzzy soft mapping. Then fuzzy soft function $f_{p u}: F(X, A) \rightarrow F(Y, B)$ is fuzzy soft continuous, if for any fuzzy soft open set $g_{A}$ in $\left(Y, \tau_{2}, B\right), f_{p u}^{-1}\left(g_{A}\right)$ is fuzzy soft open in $\left(X, \tau_{1}, A\right)$.

Definition 2.24[30]. Let $\left(X, \tau_{1}, A\right)$ and $\left(Y, \tau_{2}, B\right)$ are fuzzy soft topological spaces and $f_{p u}: F(X, A) \rightarrow F(Y, B)$ be a fuzzy soft function. Then $f_{p u}$ is said to be fuzzy soft open(resp. fuzzy soft closed), if for any fuzzy soft open (resp. fuzzy soft closed) set $h_{A}$ in $\left(X, \tau_{1}, A\right), f_{p u}\left(h_{A}\right)$ is fuzzy soft open(resp. fuzzy soft closed) in $\left(Y, \tau_{2}, B\right)$.

\section{Fuzzy soft almost soft continuous function}

Definition 3.1[18]. Let $f_{A}$ be a fuzzy soft set in fuzzy soft topological space $(X, \tau, A)$ over $X$. Then $f_{A}$ is said to be fuzzy soft regular open set over $X$, if $\left(\overline{f_{A}}\right)^{\circ} \stackrel{\sim}{=} f_{A}$.

Definition 3.2[18]. Let $f_{A}$ be a fuzzy soft set in fuzzy soft topological space $(X, \tau, A)$ over $X$. Then $f_{A}$ is said to be fuzzy soft regular closed set over $X$, if $\overline{\left(f_{A}\right)^{\circ}} \stackrel{\sim}{=} f_{A}$.

Theorem 3.3[18] (1) $f_{A}$ is said to be fuzzy soft regular open set over $X$ if and only if $\left(f_{A}\right)^{c}$ is said to be fuzzy soft regular closed set over $X$.

(2) Every fuzzy soft regular open (closed) set is a fuzzy soft open(closed) set.

Now we define:

Definition 3.4. Let $\left(X, \tau_{1}, A\right)$ and $\left(Y, \tau_{2}, B\right)$ be two fuzzy soft topological spaces and $f_{p u}: F(X, A) \rightarrow F(Y, B)$ be a fuzzy soft mapping. Then $f_{p u}$ is said to be fuzzy soft almost soft continuous, if for any fuzzy soft regular-open set $g_{B}$ in $\left(Y, \tau_{2}, B\right), f_{p u}^{-1}\left(g_{B}\right)$ is fuzzy soft open in $\left(X, \tau_{1}, A\right)$.

Example 3.5. Let $X=\left\{h_{1}, h_{2}, h_{3}\right\}, Y=\left\{x_{1}, x_{2}, x_{3}\right\}, A=\left\{e_{1}, e_{2}\right\}, B=\left\{e_{1}^{\prime}, e_{2}^{\prime}\right\}$ and $\tau=\left\{\tilde{0}, \tilde{1},\left(f_{A}\right)_{1},\left(f_{A}\right)_{2},\left(f_{A}\right)_{3},\left(f_{A}\right)_{4}\right\}$, $\tau^{*}=\left\{\tilde{0}, \tilde{1},\left(g_{B}\right)_{1},\left(g_{B}\right)_{2}\right\}$. Where $\left(f_{A}\right)_{1},\left(f_{A}\right)_{2},\left(f_{A}\right)_{3},\left(f_{A}\right)_{4}$ are fuzzy soft sets over $X$ and $\left(g_{B}\right)_{1},\left(g_{B}\right)_{2}$ are fuzzy soft sets over $Y$, defined as follows

$f_{1}\left(e_{1}\right)\left(h_{1}\right)=0.5, f_{1}\left(e_{1}\right)\left(h_{2}\right)=0.3, f_{1}\left(e_{1}\right)\left(h_{3}\right)=0.2$,

$f_{1}\left(e_{2}\right)\left(h_{1}\right)=0.3, f_{1}\left(e_{2}\right)\left(h_{2}\right)=0.5, f_{1}\left(e_{2}\right)\left(h_{3}\right)=0.2$,

$f_{2}\left(e_{1}\right)\left(h_{1}\right)=1, f_{2}\left(e_{1}\right)\left(h_{2}\right)=0, f_{2}\left(e_{1}\right)\left(h_{3}\right)=0.5$,

$f_{2}\left(e_{2}\right)\left(h_{1}\right)=0.5, f_{2}\left(e_{2}\right)\left(h_{2}\right)=0.3, f_{2}\left(e_{2}\right)\left(h_{3}\right)=1$,

$f_{3}\left(e_{1}\right)\left(h_{1}\right)=0.5, f_{3}\left(e_{1}\right)\left(h_{2}\right)=0, f_{3}\left(e_{1}\right)\left(h_{3}\right)=0.2$,

$f_{3}\left(e_{2}\right)\left(h_{1}\right)=0.3, f_{3}\left(e_{2}\right)\left(h_{2}\right)=0.3, f_{3}\left(e_{2}\right)\left(h_{3}\right)=0.2$,

$f_{4}\left(e_{1}\right)\left(h_{1}\right)=1, f_{4}\left(e_{1}\right)\left(h_{2}\right)=0.3, f_{4}\left(e_{1}\right)\left(h_{3}\right)=0.5$,

$f_{4}\left(e_{2}\right)\left(h_{1}\right)=0.5, f_{4}\left(e_{2}\right)\left(h_{2}\right)=0.5, f_{4}\left(e_{2}\right)\left(h_{3}\right)=1$.

and

$g_{1}\left(e_{1}^{\prime}\right)\left(x_{1}\right)=0.2, g_{1}\left(e_{1}^{\prime}\right)\left(x_{2}\right)=0.3, g_{1}\left(e_{1}^{\prime}\right)\left(x_{3}\right)=0.5$,

$g_{1}\left(e_{2}^{\prime}\right)\left(x_{1}\right)=0.2, g_{1}\left(e_{2}^{\prime}\right)\left(x_{2}\right)=0.5, g_{1}\left(e_{2}^{\prime}\right)\left(x_{3}\right)=0.3$,

$g_{2}\left(e_{1}^{\prime}\right)\left(x_{1}\right)=0.8, g_{2}\left(e_{1}^{\prime}\right)\left(x_{2}\right)=0.7, g_{2}\left(e_{1}^{\prime}\right)\left(x_{3}\right)=0.5$,

$g_{2}\left(e_{2}\right)\left(x_{1}\right)=0.8, g_{2}\left(e_{2}\right)\left(x_{2}\right)=0.5, g_{2}\left(e_{2}\right)\left(x_{3}\right)=0.7$.

Then $\tau$ and $\tau^{*}$ are fuzzy soft topologies on $X$ and $Y$ respectively. Therefore, $(X, \tau, A)$ and $\left(Y, \tau^{*}, B\right)$ are fuzzy soft topological spaces over $X$ and $Y$ respectively. Note that the fuzzy soft closed sets in $\left(Y, \tau^{*}, B\right)$ are $\left\{\left\{x_{0.8}, x_{0.7}, x_{0.5}\right\},\left\{x_{0.8}, x_{0.5}, x_{0.7}\right\}\right\},\left\{\left\{x_{0.2}, x_{0.3}, x_{0.5}\right\},\left\{x_{0.2}, x_{0.5}, x_{0.3}\right\}\right\}, \tilde{1}$ and $\tilde{0}$.

Let us take fuzzy soft set $g_{B}$ over $Y$ defined by 
$g\left(e_{1}^{\prime}\right)\left(x_{1}\right)=0.2, g\left(e_{1}^{\prime}\right)\left(x_{2}\right)=0.3, g\left(e_{1}^{\prime}\right)\left(x_{3}\right)=0.5$,

$g\left(e_{2}^{\prime}\right)\left(x_{1}\right)=0.8, g\left(e_{2}^{\prime}\right)\left(x_{2}\right)=0.7, g\left(e_{2}^{\prime}\right)\left(x_{3}\right)=0.5$.

That is, $g_{B}=\left\{\left\{x_{0.2}, x_{0.3}, x_{0.5}\right\},\left\{x_{0.8}, x_{0.5}, x_{0.1}\right\}\right\}$. Calculation shows that $\left(\overline{g_{B}}\right)^{\circ} \stackrel{\sim}{=} g_{B}$, which implies that $g_{B}$ is fuzzy soft regular-open set.

Let us define the fuzzy soft mapping $f_{p u}: F(X, A) \rightarrow F(Y, B)$ by

$u\left(h_{1}\right)=x_{3}, u\left(h_{2}\right)=x_{2}, u\left(h_{3}\right)=x_{1}$ and $p\left(e_{1}\right)=e_{1}^{\prime}, p\left(e_{2}\right)=e_{2}^{\prime}$.

Now $f_{p u}^{-1}\left(g_{B}\right)\left(e_{1}\right) h_{1}=g\left(p\left(e_{1}\right)\right)\left(u\left(h_{1}\right)=g\left(e_{1}^{\prime}\right) x_{3}=\left\{x_{0.2}, x_{0.3}, x_{0.5}\right\} x_{3}=x_{0.5}\right.$. Similarly, $f_{p u}^{-1}\left(g_{B}\right)\left(e_{1}\right) h_{2}=x_{0.3}$ and $f_{p u}^{-1}\left(g_{B}\right)\left(e_{1}\right) h_{3}=x_{0.2}$. Also

$f_{p u}^{-1}\left(g_{B}\right)\left(e_{2}\right) h_{1}=g\left(p\left(e_{2}\right)\left(u\left(h_{1}\right)=g\left(e_{2}^{\prime}\right) x_{3}=\left\{x_{0.2}, x_{0.5}, x_{0.3}\right\} x_{3}=x_{0.3}\right.\right.$.

Similarly, $f_{p u}^{-1}\left(g_{B}\right)\left(e_{2}\right) h_{2}=x_{0.5}$ and $f_{p u}^{-1}\left(g_{B}\right)\left(e_{2}\right) h_{3}=x_{0.2}$. Thus

$f_{p u}^{-1}\left(g_{B}\right) \stackrel{\sim}{=}\left\{e_{1}=\left\{\left\{x_{0.5}, x_{0.3}, x_{0.2}\right\}\right\}, e_{2}=\left\{\left\{x_{0.3}, x_{0.5}, x_{0.2}\right\}\right\}\right\}$, which is fuzzy soft open in $F(X, A)$. Thus fuzzy soft mapping $f_{p u}$ is fuzzy soft almost soft continuous, because fuzzy soft set $g_{B}$ is fuzzy soft regular-open in $F(Y, B)$ and $f_{p u}^{-1}\left(g_{B}\right)$ is fuzzy soft open in $F(X, A)$.

Theorem 3.6. Let $\left(X, \tau_{1}, A\right)$ and $\left(Y, \tau_{2}, B\right)$ be two fuzzy soft topological spaces and $f_{p u}: F(X, A) \rightarrow F(Y, B)$ be a fuzzy soft mapping. Then the following statements are equivalent:

(1) $f_{p u}$ is fuzzy soft almost soft continuous mapping.

(2) For any fuzzy soft regular-closed set $g_{B}$ in $\left(Y, \tau_{2}, B\right), f_{p u}^{-1}\left(g_{B}\right)$ is fuzzy soft closed in $\left(X, \tau_{1}, A\right)$.

(3) For any fuzzy soft open set $h_{B}$ in $\left(Y, \tau_{2}, B\right), f_{p u}^{-1}\left(h_{B}\right) \tilde{\leq}\left(f_{p u}^{-1}\left(\left(\overline{h_{B}}\right)^{\circ}\right)\right)^{\circ}$.

(4) For any fuzzy soft closed set $k_{B}$ in $\left(Y, \tau_{2}, B\right), \overline{f_{p u}^{-1}\left\{\overline{\left(k_{B}\right)^{\circ}}\right\}} \tilde{\leq} f_{p u}^{-1}\left(k_{B}\right)$.

Proof. $(1) \Leftrightarrow(2)$ This directly follows from Theorem 3.3.

$(1) \Rightarrow(3)$ Let $h_{B}$ be fuzzy soft open in $\left(Y, \tau_{2}, B\right)$. Then $h_{B} \tilde{\leq}\left(\overline{h_{B}}\right)^{\circ}$. Therefore, $f_{p u}^{-1}\left(h_{B}\right) \tilde{\leq} f_{p u}^{-1}\left(\left(\overline{h_{B}}\right)^{\circ}\right)$. By Using Theorem 5.9(b)[18], we have that $\left(\overline{h_{B}}\right)^{\circ}$ is a fuzzy soft regular-open set in $\left(Y, \tau_{2}, B\right)$. Since $f_{p u}$ is fuzzy soft almost soft continuous mapping, then $f_{p u}^{-1}\left(\left(\overline{h_{B}}\right)^{\circ}\right)$ is fuzzy soft open set in $\left(X, \tau_{1}, A\right)$. Hence, $f_{p u}^{-1}\left(h_{B}\right) \tilde{\leq} f_{p u}^{-1}\left(\left(\overline{h_{B}}\right)^{\circ}\right) \stackrel{\sim}{=}\left(f_{p u}^{-1}\left(\left(\overline{h_{B}}\right)^{\circ}\right)\right)^{\circ}$. $(3) \Rightarrow(1)$ Let $h_{B}$ be fuzzy soft regular-open in $\left(Y, \tau_{2}, B\right)$. Then

$f_{p u}^{-1}\left(h_{B}\right) \tilde{\leq}\left(f_{p u}^{-1}\left(\left(\overline{h_{B}}\right)^{\circ}\right)\right)^{\circ} \stackrel{\sim}{=}\left(f_{p u}^{-1}\left(h_{B}\right)\right)^{\circ}$. This implies that $f_{p u}^{-1}\left(h_{B}\right) \stackrel{\sim}{=}\left(f_{p u}^{-1}\left(h_{B}\right)\right)^{\circ}$. Hence $f_{p u}^{-1}\left(h_{B}\right)$ is fuzzy soft open set in $\left(X, \tau_{1}, A\right)$.

$(2) \Leftrightarrow(4)$ This can be proved in similar way.

This completes the proof.

Remark 3.7. (1) Fuzzy soft semi-continuous mapping and fuzzy soft almost soft continuous mappings are independent notions.

(2) If $f_{p u}$ is fuzzy soft almost soft continuous mapping then $f_{p u}$ is fuzzy soft continuous mapping, since every fuzzy soft regular-open set is fuzzy soft open but the converse is not true in general[18].

Definition 3.8. A fuzzy soft topological space $(X, \tau, A)$ is said to be fuzzy soft semi-regular space, if the collection of all fuzzy soft regular-open sets of $X$ forms a fuzzy soft base for fuzzy soft topological space $(X, \tau, A)$.

The proof of the following lemma follows by using Theorems 3.9 and 3.11[30].

Lemma 3.9. Let $(X, \tau, A)$ be fuzzy soft topological space and $f_{A_{i}}$ be a collections of fuzzy soft sets in $(X, \tau, A)$.

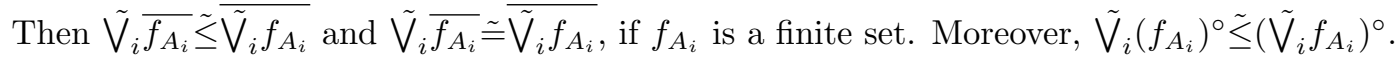

The following theorem shows that the converse of Remark $3.7(2)$ is true, if $\left(Y, \tau_{2}, B\right)$ is fuzzy soft semi-regular space.

Theorem 3.10. Let $\left(X, \tau_{1}, A\right)$ be fuzzy soft topological space, $\left(Y, \tau_{2}, B\right)$ be a fuzzy soft semi-regular space and $f_{p u}: F(X, A) \rightarrow F(Y, B)$ be a fuzzy soft mapping. Then $f_{p u}$ is fuzzy soft almost soft continuous if and only if $f_{p u}$ is fuzzy soft continuous.

Proof. Using Remark 3.7(2), it is sufficient to prove that if $f_{p u}$ is fuzzy soft almost soft continuous then it is fuzzy soft continuous. Suppose $h_{B}$ be fuzzy soft open in $\left(Y, \tau_{2}, B\right)$. Then $h_{B} \tilde{=} \tilde{\bigvee} h_{B_{\alpha}}$, where each $h_{B_{\alpha}}$ is fuzzy soft regular-open set in $\left(Y, \tau_{2}, B\right)$. Now using Theorem 3.10(3)[1], Lemma 3.9 and Theorem 3.6(3), we have

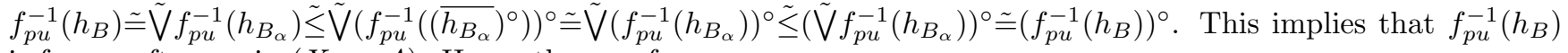
is fuzzy soft open in $\left(X, \tau_{1}, A\right)$. Hence the proof. 
Theorem 3.11. Let $f_{A}$ be a fuzzy soft set in fuzzy soft topological space $(X, \tau, A)$ over $X$. If $f_{A}$ is fuzzy soft semi-pre-open, then $\overline{f_{A}}$ is fuzzy soft regular-closed.

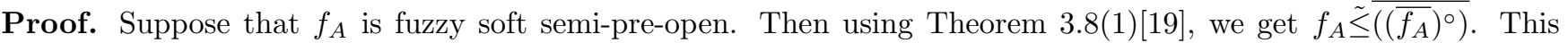

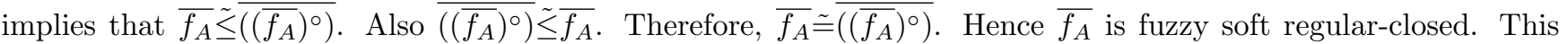
completes the proof.

Theorem 3.12. Let $\left(X, \tau_{1}, A\right)$ and $\left(Y, \tau_{2}, B\right)$ are fuzzy soft topological spaces and $f_{p u}: F(X, A) \rightarrow F(Y, B)$ be a fuzzy soft mapping. Then the following statements are equivalent to each other.

(1) $f_{p u}$ is fuzzy soft almost soft continuous.

(2) For every fuzzy soft semi-pre-open set $g_{B}$ in $\left(Y, \tau_{2}, B\right), \overline{f_{p u}^{-1}\left(g_{B}\right)} \tilde{\leq} f_{p u}^{-1}\left(\overline{g_{B}}\right)$.

(3) For every fuzzy soft semi-open set $g_{B}$ in $\left(Y, \tau_{2}, B\right), \overline{f_{p u}^{-1}\left(g_{B}\right)} \tilde{\leq} f_{p u}^{-1}\left(\overline{g_{B}}\right)$.

(4) For every fuzzy soft pre-open set $g_{B}$ in $\left(Y, \tau_{2}, B\right), f_{p u}^{-1}\left(g_{B}\right) \tilde{\leq}\left(f_{p u}^{-1}\left(\left(\overline{g_{B}}\right)^{\circ}\right)\right)^{\circ}$.

Proof. $(1) \Rightarrow(2)$ Suppose that $g_{B}$ be fuzzy soft semi-pre-open set in $\left(Y, \tau_{2}, B\right)$. Then by Theorem 3.11 , we have $\overline{g_{B}}$ is fuzzy soft regular-closed in $\left(Y, \tau_{2}, B\right)$. Since $f_{p u}$ is fuzzy soft almost soft continuous, then Theorem 3.6 implies that $f_{p u}^{-1}\left(\overline{g_{B}}\right)$ is fuzzy soft closed in $\left(X, \tau_{1}, A\right)$. Therefore, $\overline{f_{p u}^{-1}\left(g_{B}\right)} \tilde{\leq} f_{p u}^{-1}\left(\overline{g_{B}}\right)$.

$(2) \Rightarrow(3)$ This directly follows using Remark 2.20 that every fuzzy soft semi-open set is fuzzy soft semi-pre-open.

$(3) \Rightarrow(1)$ Suppose $g_{B}$ be a fuzzy soft regular closed set in $\left(Y, \tau_{2}, B\right)$. Then $g_{B} \tilde{=} \overline{\left(\left(g_{B}\right)^{\circ}\right)}$. Theorem 3.3(2) implies that $g_{B}$ is fuzzy soft semi-open set in $\left(Y, \tau_{2}, B\right)$. This follows that $\overline{f_{p u}^{-1}\left(g_{B}\right)} \tilde{\leq} f_{p u}^{-1}\left(\overline{g_{B}}\right) \tilde{=} f_{p u}^{-1}\left(g_{B}\right)$. Therefore, $f_{p u}^{-1}\left(g_{B}\right)$ is fuzzy soft closed. Hence by Theorem 3.6, $f_{p u}$ is fuzzy soft almost soft continuous.

$(1) \Rightarrow(4)$ Suppose that $g_{B}$ be fuzzy soft pre-open set in $\left(Y, \tau_{2}, B\right)$. Then $g_{B} \tilde{\leq}\left(\overline{g_{B}}\right)^{\circ}$ and $\left(\overline{g_{B}}\right)^{\circ}$ is fuzzy soft regularopen. Since $f_{p u}$ is fuzzy soft almost soft continuous, then by Theorem $3.6, f_{p u}^{-1}\left(\left(\overline{g_{B}}\right)^{\circ}\right)$ is fuzzy soft open set in $\left(X, \tau_{1}, A\right)$. Therefore, $f_{p u}^{-1}\left(g_{B}\right) \tilde{\leq} f_{p u}^{-1}\left(\left(\overline{g_{B}}\right)^{\circ}\right) \tilde{=}\left(f_{p u}^{-1}\left(\overline{g_{B}}\right)^{\circ}\right)^{\circ}$.

$(4) \Rightarrow(1)$ Suppose that $g_{B}$ be fuzzy soft regular-open set in $\left(Y, \tau_{2}, B\right)$. Then $g_{B}$ is fuzzy soft pre-open set in

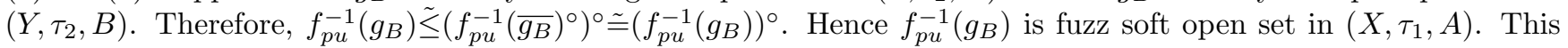
follows that $f_{p u}$ is fuzzy soft almost soft continuous function. Hence the proof.

The following lemma directly follows from definition of fuzzy soft pre-closure $F^{s} p c l^{s}$ of the fuzzy soft set $f_{A}$.

Lemma 3.13. Let $e\left(f_{A}\right)$ be a fuzzy soft point in a fuzzy soft topological space $(X, \tau, A)$. Then $e\left(f_{A}\right) \tilde{\in} F^{s} p c l^{s}\left(h_{A}\right)$ if and only if for any fuzzy soft pre-open set $k_{A}$ in $(X, \tau, A)$ with $e\left(f_{A}\right) \tilde{\epsilon} k_{A}$ implies $h_{A} \tilde{\wedge} k_{A} \tilde{\neq} \tilde{\phi}$.

Theorem 3.14. Let $(X, \tau, A)$ be fuzzy soft topological space and $f_{A}$ is fuzzy soft semi-open set in $(X, \tau, A)$. Then $F^{s} p c l^{s}\left(f_{A}\right) \tilde{=} \overline{f_{A}}$.

Proof. For any fuzzy soft set $f_{A}, F^{s} p c l^{s}\left(f_{A}\right) \tilde{\leq} \overline{f_{A}}$ is obviously true. Thus it is enough to show that $\overline{f_{A}} \tilde{\leq} F^{s} p c l^{s}\left(f_{A}\right)$, for fuzzy soft semi-open set $f_{A}$ in $(X, \tau, A)$. For this, let $e\left(g_{A}\right) \tilde{\epsilon} \overline{f_{A}}$ and $e\left(g_{A}\right) \tilde{\epsilon} k_{A}$, where $k_{A}$ is fuzzy soft preopen set in $(X, \tau, A)$. Then $e\left(g_{A}\right) \tilde{\in} k_{A} \tilde{\in}\left(\overline{k_{A}}\right)^{\circ}$ and hence $f_{A} \tilde{\Lambda}\left(\overline{k_{A}}\right)^{\circ} \tilde{\neq} \tilde{\phi}$. Now $f_{A}$ is fuzzy soft semi-open follows that $f_{A} \tilde{\Lambda}\left(\overline{k_{A}}\right)^{\circ} \tilde{\leq} \overline{\left(\left(f_{A}\right)^{\circ}\right)} \tilde{\Lambda}\left(\overline{k_{A}}\right)^{\circ} \tilde{\leq} \overline{\left(\left(f_{A}\right)^{\circ} \tilde{\Lambda} \overline{k_{A}}\right)} \tilde{\leq} \overline{\left(f_{A} \tilde{\Lambda} k_{A}\right)}$. Hence, we get $\overline{\left(f_{A} \tilde{\Lambda} k_{A}\right)} \tilde{\neq} \tilde{\phi}$. This implies that $f_{A} \tilde{\Lambda} k_{A} \tilde{\neq} \tilde{\phi}$. Using Lemma 3.13, we get $e\left(g_{A}\right) \tilde{\in} F^{s} p c l^{s}\left(f_{A}\right)$. Therefore, $\overline{f_{A}} \tilde{\leq} F^{s} p c l^{s}\left(f_{A}\right)$. This completes the proof.

The proof of the following theorem follows in similar lines as in Theorem 3.12 and is therefore omitted.

Theorem 3.15. Let $\left(X, \tau_{1}, A\right)$ and $\left(Y, \tau_{2}, B\right)$ are fuzzy soft topological spaces and $f_{p u}: F(X, A) \rightarrow F(Y, B)$ be a fuzzy soft mapping. Then the following statements are equivalent to each other.

(1) $f_{p u}$ is fuzzy soft almost soft continuous.

(2) For every fuzzy soft semi-pre-open set $g_{B}$ in $\left(Y, \tau_{2}, B\right), \overline{f_{p u}^{-1}\left(g_{B}\right)} \tilde{\leq} f_{p u}^{-1}\left(F^{s} c l^{\alpha}\left(g_{B}\right)\right)$.

(3) For every fuzzy soft semi-open set $g_{B}$ in $\left(Y, \tau_{2}, B\right), \overline{f_{p u}^{-1}\left(g_{B}\right)} \tilde{\leq} f_{p u}^{-1}\left(F^{s} p c l^{s}\left(g_{B}\right)\right)$.

(4) For every fuzzy soft pre-open set $g_{B}$ in $\left(Y, \tau_{2}, B\right), f_{p u}^{-1}\left(g_{B}\right) \tilde{\leq}\left(f_{p u}^{-1}\left(\operatorname{scl}^{f s}\left(g_{B}\right)\right)\right)^{\circ}$.

Definition 3.16. Let $\left(X, \tau_{1}, A\right)$ and $\left(Y, \tau_{2}, B\right)$ are fuzzy soft topological spaces and $f_{p u}: F(X, A) \rightarrow F(Y, B)$ be a fuzzy soft mapping. Then $f_{p u}$ is said to be fuzzy soft almost soft open(resp. closed), if for any fuzzy soft regular-open (resp. closed) set $h_{A}$ in $\left(X, \tau_{1}, A\right), f_{p u}\left(h_{A}\right)$ is fuzzy soft open(resp. closed) in $\left(Y, \tau_{2}, B\right)$.

Definition 3.17. Let $\left(X, \tau_{1}, A\right)$ and $\left(Y, \tau_{2}, B\right)$ are fuzzy soft topological spaces and $f_{p u}: F(X, A) \rightarrow F(Y, B)$ be a 
fuzzy soft mapping. Then fuzzy soft surjective fuzzy soft mapping $f_{p u}: F(X, A) \rightarrow F(Y, B)$ is fuzzy soft almost soft quasi-compact if and only if $f_{p u}^{-1}\left(g_{A}\right)$ is fuzzy soft regular-open in $\left(X, \tau_{1}, A\right)$ follows $g_{A}$ is fuzzy soft open in $\left(Y, \tau_{2}, B\right)$.

The proof of the following theorem directly follows from the above Definition 3.17.

Theorem 3.18. Let $\left(X, \tau_{1}, A\right)$ and $\left(Y, \tau_{2}, B\right)$ are fuzzy soft topological spaces and $f_{p u}: F(X, A) \rightarrow F(Y, B)$ be a fuzzy soft mapping. Then the following statements are equivalent:

(1) $f_{p u}$ is fuzzy soft bijective and fuzzy soft almost soft quasi-compact.

(2) The fuzzy soft inverse image of every fuzzy soft regular-open(resp. closed) set is fuzzy soft open(resp. closed).

Now we establish the following characterizations of fuzzy soft almost soft open functions as:

Theorem 3.19. Let $\left(X, \tau_{1}, A\right)$ and $\left(Y, \tau_{2}, B\right)$ are fuzzy soft topological spaces and $f_{p u}: F(X, A) \rightarrow F(Y, B)$ be a fuzzy soft bijective mapping. Then the following statements are equivalent:

(1) $f_{p u}$ is fuzzy soft almost soft open.

(2) $f_{p u}$ is fuzzy soft almost soft closed.

(3) $f_{p u}$ is fuzzy soft almost soft quasi-compact.

(4) $f_{p u}^{-1}$ is fuzzy soft almost soft continuous.

Proof. $(1) \Rightarrow(2)$ Suppose $f_{A}$ be fuzzy soft regular-closed in $\left(X, \tau_{1}, A\right)$. Then $\left(f_{A}\right)^{c}$ be fuzzy soft regular-open. Since $f_{p u}$ is fuzzy soft almost soft open, then $f_{p u}\left(\left(f_{A}\right)^{c}\right)$ is fuzzy soft open. This implies that $\left(f_{p u}\left(f_{A}\right)\right)^{c}$ is fuzzy soft open. Hence $f_{p u}\left(f_{A}\right)$ is fuzzy soft closed. Therefore, $f_{p u}$ is fuzzy soft almost soft closed.

$(2) \Rightarrow(3)$ Suppose that $f_{p u}^{-1}\left(h_{A}\right)$ is fuzzy soft regular-closed. Then Theorem 3.18 implies that $f_{p u} f_{p u}^{-1}\left(h_{A}\right)$ is fuzzy soft closed. This follows that $h_{A}$ is fuzzy soft closed. Therefore, $f_{p u}$ is fuzzy soft almost soft quasi-compact.

$(3) \Rightarrow(4)$ Suppose that $k_{A}$ be fuzzy soft regular open in $\left(X, \tau_{1}, A\right)$. Then $f_{p u}^{-1} f_{p u}\left(k_{A}\right) \tilde{=} k_{A}$ is fuzzy soft regular-open. Therefore, $f_{p u}\left(k_{A}\right)$ is fuzzy soft open. That is, $\left(f_{p u}^{-1}\right)^{-1}\left(k_{A}\right)$ is fuzzy soft open. This follows that $f_{p u}^{-1}$ is fuzzy soft almost soft continuous.

$(4) \Rightarrow(1)$. Suppose $f_{A}$ be a fuzzy soft regular-open in $\left(X, \tau_{1}, A\right)$. Then by $(4),\left(f_{p u}^{-1}\right)^{-1}\left(f_{A}\right)$ is fuzzy soft open in $\left(Y, \tau_{2}, B\right)$. Hence $f_{p u}$ is fuzzy soft almost soft open. Hence the proof.

Theorem 3.20. Let $\left(X, \tau_{1}, A\right),\left(Y, \tau_{2}, B\right)$ and $\left(Z, \tau_{3}, C\right)$ are fuzzy soft topological spaces and $f_{p u}: F(X, A) \rightarrow$ $F(Y, B)$ be a fuzzy soft open and fuzzy soft continuous mapping, where $u: X \rightarrow Y$ and $p: A \rightarrow B$ are mappings and let $g_{q v}: F(Y, B) \rightarrow F(Z, C)$ be a fuzzy soft mapping, where $v: Y \rightarrow Z$ and $q: B \rightarrow C$ are mappings. Then $(g \circ f)_{p v}$ is fuzzy soft almost soft continuous if and only if $g_{q v}$ is fuzzy soft almost soft continuous.

Proof. $(\Rightarrow)$ Suppose that $g_{q v}$ is fuzzy soft almost soft continuous. Let $(H, C)$ be fuzzy soft regular-open subset in $\left(Z, \tau_{3}, C\right)$. This implies that

$(g \circ f)_{p v}^{-1}(H, C)$ is fuzzy soft open in $\left(X, \tau_{1}, A\right)$ and hence $f_{p u}^{-1}\left(g_{q v}^{-1}(H, C)\right)$ is fuzzy soft open in $\left(X, \tau_{1}, A\right)$. Since $f_{p u}$ is fuzzy soft open, then $f_{p u}\left(f_{p u}^{-1}\left(g_{q v}^{-1}(H, C)\right)\right)$ is fuzzy soft open in $\left(Y, \tau_{2}, B\right)$. Therefore, $g_{q v}^{-1}(H, C)$ is fuzzy soft open in $\left(Y, \tau_{2}, B\right)$. Thus $g_{q v}$ is fuzzy soft almost soft continuous.

$(\Leftarrow)$ Suppose that $g_{q v}$ is fuzzy soft almost soft continuous and let $(H, C)$ be fuzzy soft regular-open subset in $\left(Z, \tau_{3}, C\right)$. Then $g_{q v}^{-1}(H, C)$ is fuzzy soft open in $\left(Y, \tau_{2}, B\right)$. Since $f_{p u}$ is fuzzy soft continuous, then $f_{p u}^{-1}\left(g_{q v}^{-1}(H, C)\right)$ is fuzzy soft open in $\left(X, \tau_{1}, A\right)$. Therefore, $(g \circ f)_{p v}^{-1}(H, C)$ is fuzzy soft open in $\left(X, \tau_{1}, A\right)$. Hence $(g \circ f)_{p v}$ is fuzzy soft almost soft continuous. This completes the proof.

Theorem 3.21. Let $\left(X, \tau_{1}, A\right),\left(Y, \tau_{2}, B\right)$ and $\left(Z, \tau_{3}, C\right)$ are fuzzy soft topological spaces and $f_{p u}: F(X, A) \rightarrow$ $F(Y, B)$ be a fuzzy soft mapping, where $u: X \rightarrow Y$ and $p: A \rightarrow B$ are mappings and let $g_{q v}: F(Y, B) \rightarrow F(Z, C)$ be a fuzzy soft mapping, where $v: Y \rightarrow Z$ and $q: B \rightarrow C$ are mappings. If $f_{p u}$ is fuzzy soft almost soft continuous and $(g \circ f)_{p v}$ is fuzzy soft open(closed) then $g_{q v}$ is fuzzy soft almost soft open(closed).

Proof. Suppose that $f_{p u}$ is fuzzy soft almost soft continuous and $(g \circ f)_{p v}$ is fuzzy soft open(closed). Let $(G, B)$ be a fuzzy soft regular-open subset in $\left(Y, \tau_{2}, B\right)$. Then $f_{p u}^{-1}(G, B)$ is a fuzzy soft open(closed) set in $\left(X, \tau_{1}, A\right)$. Also $(g \circ f)_{p v}$ is fuzzy soft open(closed) implies that $(g \circ f)_{p v}\left(f_{p u}^{-1}(G, B)\right)$ is fuzzy soft open(closed) set in $\left(Z, \tau_{3}, C\right)$. But $(g \circ f)_{p v}\left(f_{p u}^{-1}(G, B)\right) \tilde{=} g_{q v}(G, B)$. Therefore, $g_{q v}(G, B)$ is fuzzy soft open(closed) in $\left(Z, \tau_{3}, C\right)$. Thus $g_{q v}$ is fuzzy soft almost soft open(closed). Hence the proof.

Conclusion. We initiated and explored the interesting characterizations and properties of fuzzy soft almost soft 
continuous mappings in fuzzy soft classes. It is observed that every fuzzy soft continues mapping $f_{p u}: F(X, A) \rightarrow$ $F(Y, B)$ from fuzzy soft class $F(X, A)$ to fuzzy soft class $F(Y, B)$ is fuzzy soft almost soft continuous but the converse is not true in general. The converse is true, if the underlying fuzzy soft topological spaces $\left(Y, \tau_{2}, B\right)$ is fuzzy soft semi-regular space. We also studied and discussed the notions of fuzzy soft almost soft open(closed) mappings. Moreover, we also studied the characterizations of composition of two fuzzy soft almost soft mappings. We hope that the findings in this paper will be useful for the researchers working in the fields such as fuzzy control systems, fuzzy automata, fuzzy logic, information systems and decision making problems.

\section{References}

[1] B. Ahmad, A. Kharal: Mappings on fuzzy soft classes, Advances in Fuzzy Systems, (2009), Article ID 407890, 6 Pages.

[2] B. Ahmad, S. Hussain : On some structures of soft topology, Mathematical Sciences, 6(64) (2012), 7 Pages.

[3] C. L. Chang: Fuzzy topological spaces, Journal of Mathematical Analysis and Applications, 24(1)(1968), 182-190.

[4] B. Chen: Soft semi-open sets and related properties in soft topological spaces, Applied Mathematics Information Sciences, 7(1)(2013), 287-294.

[5] B. Chen: Soft local properties of soft semi-open sets, Discrete Dynamics in Nature and Society, Vol. 2013, Article ID 298032, 6 pages.

[6] S. Du, Q. Qin, Q. Wang, and B. Li: Fuzzy description of topological relations I: a unified fuzzy 9-intersection model, Proceedings of the 1st International Conference on Advances in Natural Computation (ICNC-05), vol. 3612 of Lecture Notes in Computer Science, pp. 1261-1273, Changsha, China, August 2005.

[7] F. Feng, Y. M. Li: Soft subsets and soft product operations, Information Sciences, 232(2013), 44-5\%.

[8] F. Feng, Y. M. Li, N. Cagman: Generalized uni-int decision making scheme based on choice value soft sets, Europeon Journal of Operaion Research, 220(2012), 162-170.

[9] F. Feng, M. Akram, B. Davvaz, V. Leoreanu-Fotea: Attribute analysis of information systems based on elementry soft implications, Knowledge Based Systems, 70(2014),281-292.

[10] F. Feng, W. Pedrycz: On scalar products and decomposition theorems of fuzzy soft sets, Journal of Multi-valued Logic and Soft Computing, 25(2015), 45-80.

[11] C. Gundaz, S. Bayramov: Some results on fuzzy soft topological spaces, Mathematical Problems in Engineering, (2013), Article ID 935308, 10 Pages.

[12] S. Hussain, B. Ahmad : Some properties of soft topological spaces, Computers and Mathematics with Applications, 62(11) (2011) 4058-4067.

[13] S. Hussain, B. Ahmad : Soft separation axioms in soft topological spaces, Hacettepe Journal of Mathematics and Statistics, 44(3) (2015), 559-568.

[14] S. Hussain: A note on soft connectedness, Journal of Egyptian Mathematical Society, 23(1) (2015), 6-11.

[15] S. Hussain: On some soft functions, Mathematical Science Letters, 4(1) (2015), 55 - 61.

[16] S. Hussain: Properties of soft semi-open and soft semi-closed sets, Pensee Journal, 76(2)(2014), 133 -143.

[17] S. Hussain: On some generalized structures in fuzzy soft topological spaces, Information Science Letters, 4(3)(2015), 107-115.

[18] S. Hussain: On weak and strong forms of fuzzy soft open sets, Fuzzy Information and Engineering, 8(2016), $451-463$.

[19] S. Hussain : On fuzzy soft semi-pre-open sets and fuzzy soft semi-pre-continuous mappings, Hacettepe Journal of Mathematics and Statistics, 46(5)(2017), 851-864.

[20] A. Z. Khameneh, A. Kilicman and A. R. Salleh: Fuzzy soft boundary, Annals of Fuzzy Mathematics and Informatics, 8(5)(2014), 687-703.

[21] Z. Kong, L. Q. Gao, L. F. Wong: Comment on a fuzzy soft set theoretic approach to decision making problems, J. Comp. Appl. Math., 223 (2009), 540-542.

[22] P. K. Maji, R. Biswas, A. R. Roy: Soft set theory, Computers and Mathematics with Applications 45(4-5) (2003), 555-562.

[23] P. K. Maji, R. Biswas, A. R. Roy: Fuzzy Soft sets, J. Fuzzy Maths., 9(3) (2001), 589 602. 
[24] D. Molodtsov: Soft set theory first results, Computers and Mathematics with Applications, 37(4-5) (1999), $19-31$.

[25] D. Molodtsov, V. Y. Leonov, D. V. Kovkov: Soft sets technique and its application, Nechetkie Sistemy i Myagkie Vychisleniya, 9(1)(2006), 28-39.

[26] G. J. Nazaroff: Fuzzy topological polysystems, Journal of Mathematical Analysis and Applications, 41(1973),734-742.

[27] D. Pie, D. Miao: From soft sets to information systems, X. Hu, Q. Liu, A. Skowron, Y. Y. Lin, R. R. Yager, B. Zhang(Eds.) Proceedings of Granular Computing Vol. 2, IEEE(2005), 2005, 617-621.

[28] M. Shabir, M. Naz: On soft topological spaces, Computers and Mathematics with Applications, 61(7)(2011), $1786-1799$.

[29] B. Tanay and M. B. Kandemir: Topological structure of fuzzy soft sets, Computers and Mathematics with Applications, 61(10)(2011), 2952-295\%.

[30] B. P. Varol and H. Aygun: Fuzzy soft topology, Hacettepe Journal of Mathematics and Statistics, 41(3)(2012), $407-419$.

[31] L. A. Zadeh: Fuzzy sets, Information and Control, 8(1996),338-353. 